\title{
Receptivity to Freestream Periodic Vorticity Disturbance on a Flat Plate with an Elliptic Leading Edge
}

\author{
S. Izawa ${ }^{1 \dagger}$, H. Isawa ${ }^{1}$, Y. Nishio ${ }^{2}$ and Y. Fukunishi ${ }^{1}$ \\ ${ }^{1}$ Tohoku University, 6-6-01 Aramaki-Aoba, Sendai, 980-8579, Japan \\ ${ }^{2}$ Seikei University, 3-3-1 Kichijoji-Kitamachi, Musashino-shi, Tokyo, 180-8633, Japan \\ † Corresponding Author Email: izawa@fluid.mech.tohoku.ac.jp
}

(Received January 16, 2020; accepted May 8, 2020)

\begin{abstract}
The receptivity of the Blasius boundary layer over a semi-infinite flat plate with an elliptic leading edge and of aspect ratio five was investigated using a direct numerical solution of two-dimensional Navier-Stokes equations. The result of the computation where the slip condition is applied to the fluctuating component of velocity at the wall surface is compared with that of an ordinary computation using a nonslip condition. Another numerical experiment is performed where no vorticity fluctuation is supplied from a freestream while prerecorded values of vorticities at the wall in response to the passage of convecting fluctuations are used as the wall vorticity boundary condition. It is shown that vorticity fluctuations in the boundary layer can be classified according to their wavelengths. Waves with longer wavelengths originate from the freestream, whereas waves with shorter wavelengths close to T-S waves originate from the surface of the plate. In another numerical experiment, the slip boundary condition against the fluctuation component of vorticity is applied to the limited area of the wall surface. The aim of the study is to determine the part of the elliptic leading edge or flat plate that induces vorticity fluctuations, thereby resulting in the creation of T-S waves. The numerical results show that the contribution of vorticity fluctuations originating from the juncture is the most crucial, whereas the vorticities supplied in the elliptic leading-edge surface negatively affect the amplitude of vorticity fluctuations inside the boundary layer. And, the stagnation section did not show positive contribution.
\end{abstract}

Keywords: Boundary layer; Receptivity; Vorticity fluctuation.

\section{INTRODUCTION}

Receptivity is the process by which environ-mental disturbances in the freestream, such as acoustic waves and vorticity fluctuations, enter a boundary layer and excite the instability modes within it. A number of reviews exist regarding this subject, such as those by Nishioka and Morkovin (1986), Kerschen (1990), Reshotko (2001), Saric et al. (2002), and Reed et al. (2015). Depending on the amplitude of the external disturbance, different structures are generated in the boundary layer. For instance, two-dimensional Tollmien-Schlichting ( TS) waves initially appear in low-disturbance environments, whereas streamwise-elongated streaks become predominant in high-disturbance environments. In the former case, instability occurs at Reynolds numbers $\operatorname{Re}_{\delta^{*}}$ greater than 520, whereas in the latter case, T-S waves are bypassed and the onset of transition occurs in the subcritical regime, where the flow quickly becomes turbulent, and the transitional Reynolds number is inversely proportional to the squared turbulence intensity (Fransson et al. 2005). The wavelengths of acoustic and vorticity disturbances are typically significantly different from those of the instability waves inside a boundary layer. This discrepancy has been explained as the wavelength conversion process (Goldstein and Hultgren 1989), in which nonparallel mean flows are a dominating factor. The growth of instability waves is affected by the geometric configurations (the leading edge, discontinuities in the surface curvature, and surface in-homogeneities). Lin et al. (1992) discovered that the continuous curvature at the ellipse-flat plate juncture lowers the receptivity of a plane acoustic disturbance. The contribution of the curvature discontinuity to the receptivity constitutes almost $50 \%$ of the total receptivity. Dietz (1999) 
investigated the receptivity to a convected disturbance using the harmonic wake from a vibrating ribbon in a freestream. A T-S wave was found to be created by the interaction of this disturbance with a two-dimensional roughness element on a flat plate. Kogan et al. (2001) used the wake behind a wire as a source of steady vortices normal to the leading edge and observed three distinct regimes of wake-boundary layer interaction: linear, symmetric non-linear, and antisymmetric nonlinear.

The possible sources of vorticity fluctuations in the boundary layer are the vorticity fluctuations in the freestream intruding into the boundary layer and the vorticity supplied at the wall in response to passing velocity fluctuations. Although the former freestream mode has a broadband spectrum, it is rapidly damped out inside a sheared region, and among them only the long wave-length disturbances are able to deeply penetrate into the boundary layer (Jacobs and Durbin 1998, Hunt and Durbin 1999). The latter wall mode is thus insensitive to short wavelength disturbances of the freestream mode owing to this "shear sheltering" phenomenon. Sengupta and Sengupta (2019) performed direct numerical simulation to investigate the vortexinduced instability caused by a translating vortex at a constant speed outside a zero-pressure gradient boundary layer.

In our previous studies, Noro et al. (2013) introduced a pair of vortex rings in a freestream outside a boundary layer by ejecting jets from two nozzles facing each other and discovered that high and low speed regions aligned in the streamwise direction were generated near the wall in response to the collision of the vortex rings. Furthermore, they discovered that vortex rings directly intruded the boundary layer, triggering a transition to downstream turbulence. In the study by Noro et al. (2014), the indirect contamination of the Blasius boundary layer owing to external disturbances was modeled by applying a slip boundary condition at the wall. It was discovered that the velocity fluctuations increased rapidly only when the line vortices outside of the boundary layer were aligned in the streamwise direction. In the study by Nishio et al. (2013), the leading-edge receptivity to the freestream vortical fluctuations whose axes were normal to the flat plate surface was discussed. It was discovered that the streamwise structures inside the boundary layer were formed owing to the velocity field induced by the vortices outside the boundary layer whose axes were normal to the wall. However, owing to the complexity of the disturbed fluid motion in the near wall region, it was difficult to discuss the interaction between convecting disturbances and resulting wall-supplied vorticity fluctuations.

In this study, two-dimensional numerical simulations are performed to investigate the leading-edge receptivity to periodic spanwise vorticity fluctuations in the freestream. Several numerical experiments are performed using the slip boundary condition at the wall against the fluctuating component of the local velocity.
Additionally, a numerical experiment is performed where the prerecorded values of vorticities at the wall are used as the wall boundary condition while no vorticity fluctuation is supplied in the freestream. The main purpose of this investigation is to point out the source of the vorticity fluctuation which later develops inside the boundary layer.

\section{COMPUTATIONAL METHOD}

\subsection{Numerical Schemes and Computational Domain}

The two-dimensional incompressible Navier-Stokes equations

$$
\frac{\partial \boldsymbol{u}}{\partial t}+\boldsymbol{u} \cdot \nabla \boldsymbol{u}=-\frac{1}{\rho} \nabla p+\frac{1}{\operatorname{Re}} \nabla^{2} \boldsymbol{u},
$$

and the continuity equation

$$
\nabla \boldsymbol{u}=0,
$$

were solved by the finite difference method, where $\boldsymbol{u}$ is the velocity, $p$ the pressure, $\rho$ the density, and $R e$ the Reynolds number. All variables were nondimensionalized by the uniform velocity $U$ and the minor axis radius of the elliptic leading edge $L b$. The marker and cell method was used to calculate the velocity and pressure. Time integration was performed by applying the first-order accurate Euler implicit method to the viscous terms and the second-order accurate Adams-Bashforth method to the convection terms. The second-order accurate central-difference scheme was applied to the spatial derivatives, except for the convection terms, which were discretized by the third-order upstream difference scheme. The velocity and pressure fields were decomposed into base-flow and fluctuation components; the fluctuation components were subsequently computed to capture the velocity fluctuations with high accuracy. The base flow was a steady flow over a semi-infinite flat plate and was obtained by a preliminary computation.

The computational domain is shown in Fig. 1. A flat plate of thickness $2 L_{b}$ was placed $35 L_{b}$ from the inlet boundary in the streamwise direction. The plate had an elliptic leading edge with an aspect ratio $L_{b} / L_{a}=5$. The configuration was selected such that the induced velocity deriving from the fluctuations entering the flow field at the upstream boundary did not directly affect the tip area of the leading edge. The origin of the Cartesian coordinate sys-tem was located at the tip of the leading edge, and the $\mathrm{x}$ and $\mathrm{y}$ axes represent the streamwise and vertical directions, respectively. The body-fitted curvilinear coordinate system $(\xi, \eta)$ was used for the grid, where the grid point number was $561 \times 281$. The grids were concentrated near the plate surface, especially in the leading edge and juncture areas, and around the $x$-axis in the freestream upstream of the leading edge to prevent the significant numerical damping of the introduced vorticity fluctuations. The grid spacings downstream of the juncture were gradually widened to increase the numerical viscosity such that unfavorable numerical 
reflections from the outflow boundary could be prevented (Nishio et al. 2013). Assuming that the phase velocity of T-S waves is $35 \%$ of the freestream velocity, the wavelength of the T-S wave can be estimated as approximately $\lambda_{\mathrm{TS}}=1.75$ when the forcing frequency $\mathrm{f}$ in Eq. (3) is 0.2. Hence, one period of a T-S wave is represented by approximately 15 points in the streamwise direction at $x=7.5$ and six points at $x=15.0$. Accordingly, the discussion on the near wall fluid motion is confined to the region upstream of $x=15.0$. All simulations were performed at $\operatorname{Re}(=\mathrm{ULb} / v)=$ 10,000 .

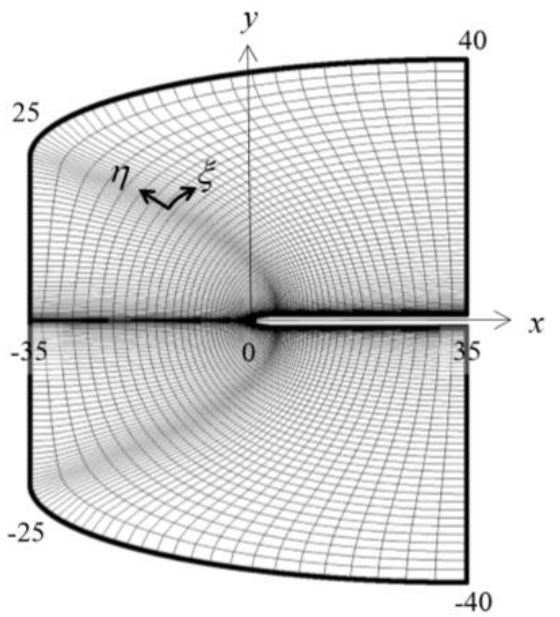

(a) Overview

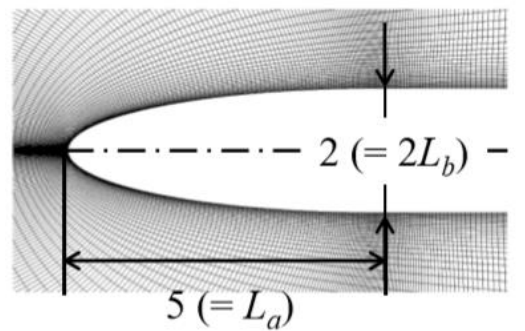

(b) Close-up view

Fig. 1. Overview and close-up view of computational domain.

\subsection{Addition of Periodic Vorticity fluctuation}

A preliminary computation was first performed to obtain the steady flow field around the plate as a base flow. The nonslip condition was imposed at the plate surface, and the Neumann boundary condition was applied at the outlet boundary. The velocity distribution of the potential flow field computed by the discrete vortex method was presented at the other outer boundaries. At the outlet boundary, the pressure was fixed to the fixed value of one, whereas the Neumann condition was applied at other boundaries. These boundary conditions were also applied during the main computations, except at the inlet boundary where periodic velocity fluctuations were added to the uniform flow. The added velocity fluctuations are expressed as
$u^{\prime}=A \phi(y) \sin (2 \pi f t)$,

$v^{\prime}=0$,

where the amplitude $\mathrm{A}$ is 0.01 , and the frequency $f$ is $0.2 ; \mathrm{t}=0$ represents the start of the vorticity fluctuation addition. A smoothing function $\phi$

$$
\phi(y)=\left(1+\frac{1}{2 \alpha}\right) \frac{r_{c}}{y}\left[1-\exp \left(-\alpha \frac{y^{2}}{r_{c}^{2}}\right)\right],
$$

was used such that the velocity fluctuations were similar to the Lamb-Oseen vortex, where the LambOseen constant $\alpha=1.25643$ and the core vortex radius $r_{c}=3$. The velocity fluctuations $u^{\prime}$ at $2 \pi f t=$ 0 and $\phi$ are shown in Fig. 2. The magnitude of $u^{\prime}$ was maximized at the outer edge of the vortex core, $y= \pm r_{c}$. The velocity fluctuations at the inlet was weak and did not directly affect the stagnation region of the plate leading edge, thereby triggering strong velocity fluctuations in the downstream boundary layer. Naturally, the streamwise scale of the vortices depends on the forcing frequency. In this simulation, the distance between the neighboring vortices with opposite signs is constant at $U / f=5$.

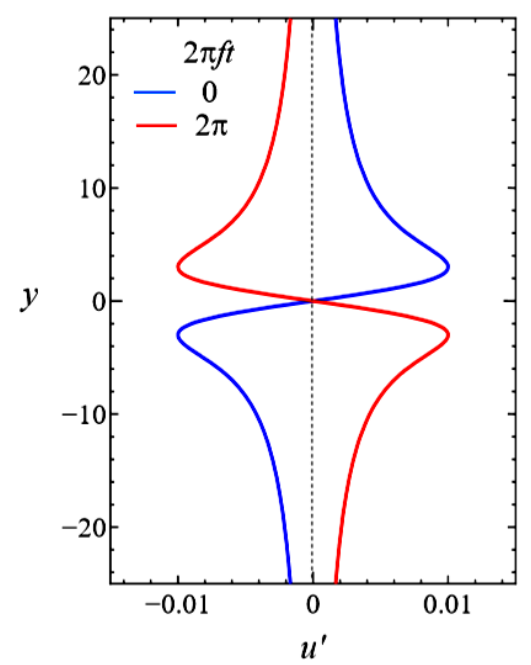

Fig. 2. Velocity fluctuations at $2 \pi f t=0$ (blue) and $\phi$ (red).

At the forcing frequency of $f=0.2$, the neutral points, i.e., branches I and II, were estimated using the linear-stability theory to be $\operatorname{Re}_{\delta^{*}} \approx 650$ and $\operatorname{Re}_{\delta^{*}} \approx 1030$, respectively. The corresponding locations were approximately $x=14.1$ and $x=35.0$. Therefore, the entire unstable region was within the flat plate region of the computational domain.

\subsection{Application of Slip Boundary Condition at Wall Surface}

To evaluate the role of the vorticity fluctuations supplied at the wall, a modified slip boundary condition was applied against the fluctuating components of the velocity in order to satisfy Eq. 5 . 
As the wall normal velocity $v_{\eta}^{\prime}$ is always zero on the wall surface, the fluctuation component of the tangential velocity at the slipping wall should satisfy

$$
\omega^{\prime}(\xi, 0)=\frac{\partial v_{\eta}^{\prime}}{\partial \xi}-\left.\frac{\partial u_{\xi}^{\prime}}{\partial \eta}\right|_{\eta=0}=-\left.\frac{\partial u_{\xi}^{\prime}}{\partial \eta}\right|_{\eta=0}=0 .
$$

The procedure generates no vorticity fluctuations at the wall. It is noteworthy that because the slip condition above was applied to only the fluctuation components, the base flow did not exhibit any change in the velocity profile.

\section{RESULTS AND DISCUSSION}

\subsection{Computation of Base flow}

The preliminary computation given by Eq. (1) was first performed to obtain the base flow, which was a steady flow field around the plate placed in a uniform flow. The resulting pressure gradient along the plate surface, shown in Fig. 3, indicates a strong favorable pressure gradient in the vicinity of the stagnation point where the flow is accelerated along the wall, followed by a quick recovery of the pressure. The pressure gradient $d p / d \xi$ changes from negative to positive at approximately $x=2.3$ and reaches the maximum of 0.035 at the junction between the elliptically curved leading edge section and the flat surface section before asymptotically approaching zero.

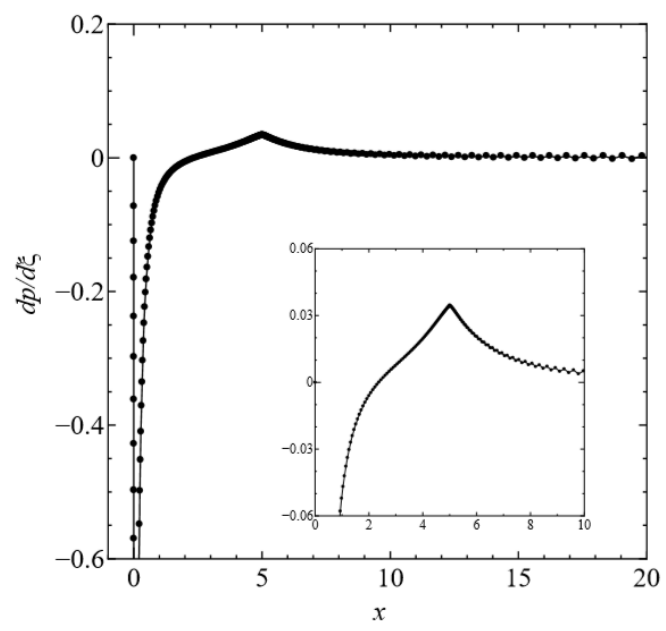

Fig. 3. Surface pressure gradient of base flow and its enlarged view focusing on juncture region.

\subsection{Effect of Freestream Vorticity fluctuation on the Boundary Layer}

Next, vorticity fluctuations were added at the inflow boundary. The distribution of vorticity fluctuations around the leading edge at an instant after a sufficiently long time is shown in Fig. 4. Here, $\omega^{\prime}$ is the instantaneous deviation of the vorticity component from the local time-averaged value. An alternate $\mathrm{x}$ direction pattern of positive and negative vorticity fluctuations was established, which gradually deformed into an arch shape owing to the flat plate. Thin and strong vorticity fluctuations appeared inside the thin boundary layer along the curved wall surface, whereas the vorticity fluctuations outside the boundary layer exhibit a wide and striped pat-tern.

Closer-range views of the distributions of vorticity fluctuations $\omega^{\prime}$ are shown in Fig. 5 at three locations: the leading edge, juncture, and flat surface. Owing to the velocity gradient inside the boundary layer, the pattern became elongated in the wall-parallel direction, and the areas of oppositesign vorticity fluctuations pile up one over another, forming a laterally striped pattern. These layers continue to elongate and become thinner while passing the leading edge area. When elongated layers whose signs are opposite are adjacent to each other and the distance between the neighboring layers decreases, viscous diffusion causes reduction in their magnitude. The vorticity fluctuations not only convect downstream but generate new vorticities at the wall surface because the nonslip boundary con-dition must be satisfied (Noro et al. 2014). Consequently, two sources exist for the vorticity fluctuations inside the boundary layer: freestream and wall surface. Therefore, the vorticity fluctuations observed in Figs. 4 and 5 can be regarded as combinations of two vorticity fluctuations originating from two different sources.

The instantaneous velocity fluctuations $u^{\prime}$ measured along the $\mathrm{x}$-direction at different distances from the wall, namely $\eta_{\mathrm{n}}=1.0,3.0$, and 4.0 , are shown in Fig. 6 . The velocity fluctuations were measured for 11 cycles. The height $\eta_{\mathrm{n}}$ represents the nondimensional distance based on the freestream velocity $U$ and the distance from the leading edge $x$,

$\eta_{n}=d_{n} \sqrt{\frac{U}{v x}}=\frac{d_{n}}{x} \sqrt{\operatorname{Re}_{x}}$,

where the dn is the wall-normal distance. Here, the height of $\eta_{\mathrm{n}}=5.0$ corresponds to the outer edge of the boundary layer, and Rex is the Reynolds number based on the streamwise coordinate, $x$.

Spatially periodic oscillations were observed in both the freestream and near-wall regions. The frequencies of these oscillations are the same everywhere, $f=0.2$, which is the frequency of the vorticity fluctuations convecting outside the boundary layer. The differences in their wavelengths de-rive from the differences in their convecting velocities. For example, in case of the velocity fluctuation measured at $\eta_{\mathrm{n}}=1.0$, the wavelength $\lambda$ is approximately 1.7 . So, its phase velocity is approximately $0.34 \%$ of the freestream velocity, which coincides with the phase velocity of T-S waves. This indicates that it is a T-S wave. Meanwhile, the phase velocity of the wave measured at $\eta_{\mathrm{n}}=5.0$ is similar to the convecting velocity of the vorticity fluctuations in the freestream. The velocity fluctuation measured at $\eta_{\mathrm{n}}$ $=4.0$ appears to be a mixture of the two waves above. 


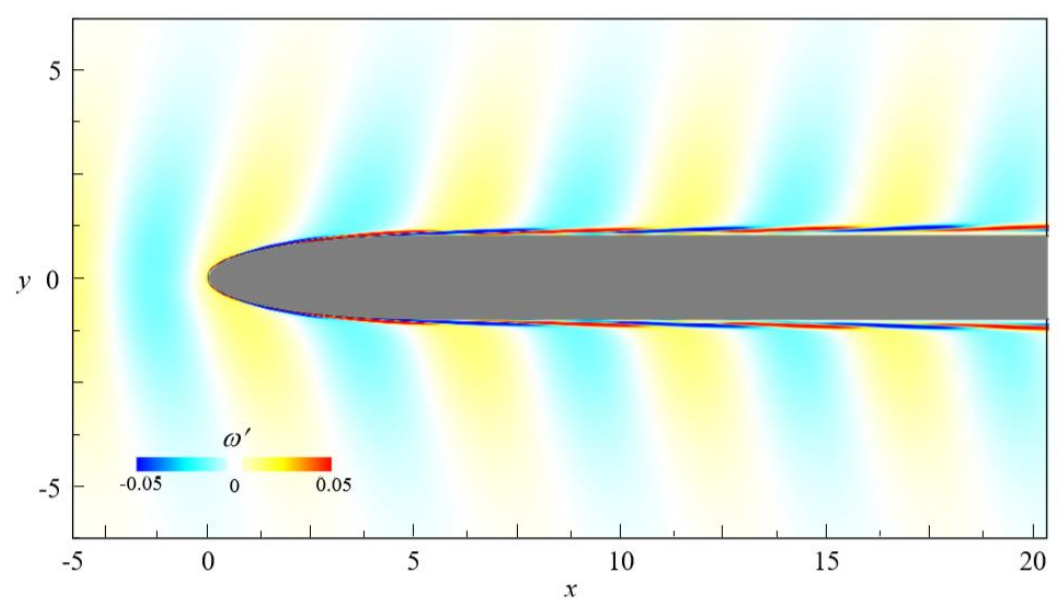

Fig.4. Instantaneous distribution of the velocity fluctuation $\omega^{\prime}$ around the leading edge.

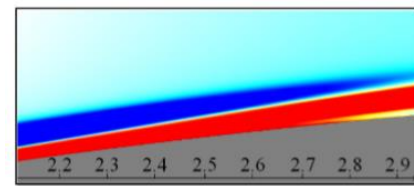

(a) Leading-edge

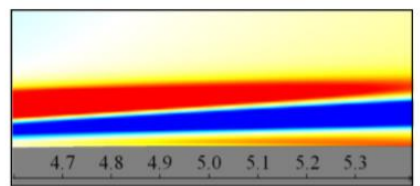

(b) Juncture

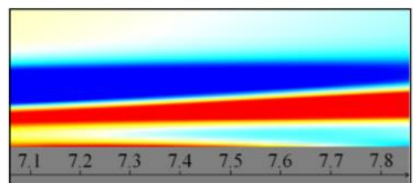

(c) Flat surface

Fig. 5. Enlarged views of Fig. 4 at three locations.

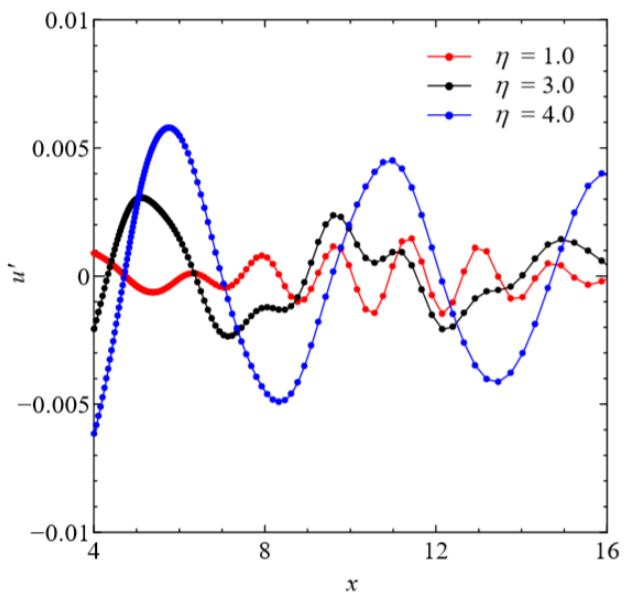

Fig. 6. Instantaneous streamwise velocity fluctuations $u^{\prime}$ at $\eta_{\mathrm{n}}=1.0,3.0,4.0$ and $\mathrm{t}=125$ for nonslip condition.
The two waves were separated by applying the Lomb-Scargle (VanderPlas 2018) algorithm. The method was chosen because it was suitable for handling data which were sampled unevenly in space. Data from the streamwise range of $7.0 \leq x \leq$ 16.0 was used in the analysis. The results are presented in Fig. 7, where the abscissa is the periodogram peak normalized by the freestream value. As shown, the T-S wave is dominant near the wall until approximately $\eta_{\mathrm{n}}=2.5$, while away from the wall, the velocity fluctuations are convecting down-stream at the freestream velocity.

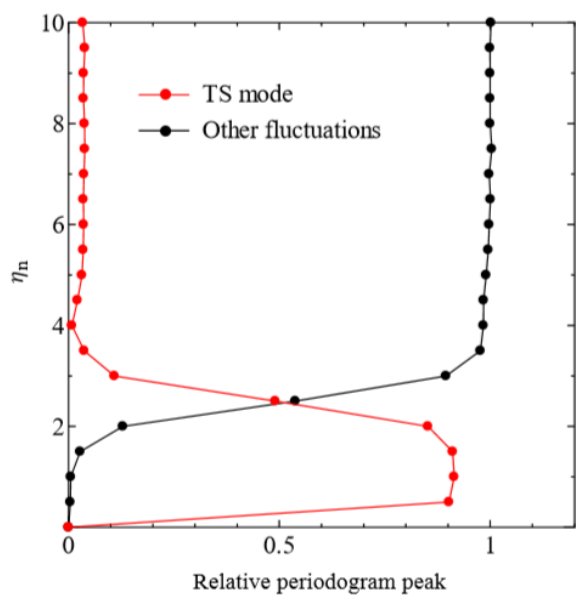

Fig. 7. Comparison of computed peaks of the Lomb-Scargle periodogram for fluctuations convecting at freestream velocity and T-S mode. Data of $x=7.0 \sim 16.0$ and $t=125$ under nonslip conditions is used.

\subsection{Slip Boundary Condition Applied at all Surfaces}

Next, the slip boundary condition was applied at the surface for the fluctuating components of the velocities. The root-mean-square (RMS) of the vortic-ity fluctuations $\omega_{\text {rms }}^{\prime}$ at different $x$-stations are presented in Fig. 8. The fluctuating component of the vorticity is always zero at the wall in the slip boundary condition case because no vorticity fluctuation is supplied from the wall. Therefore, all the vorticity fluctuations inside the boundary layer 

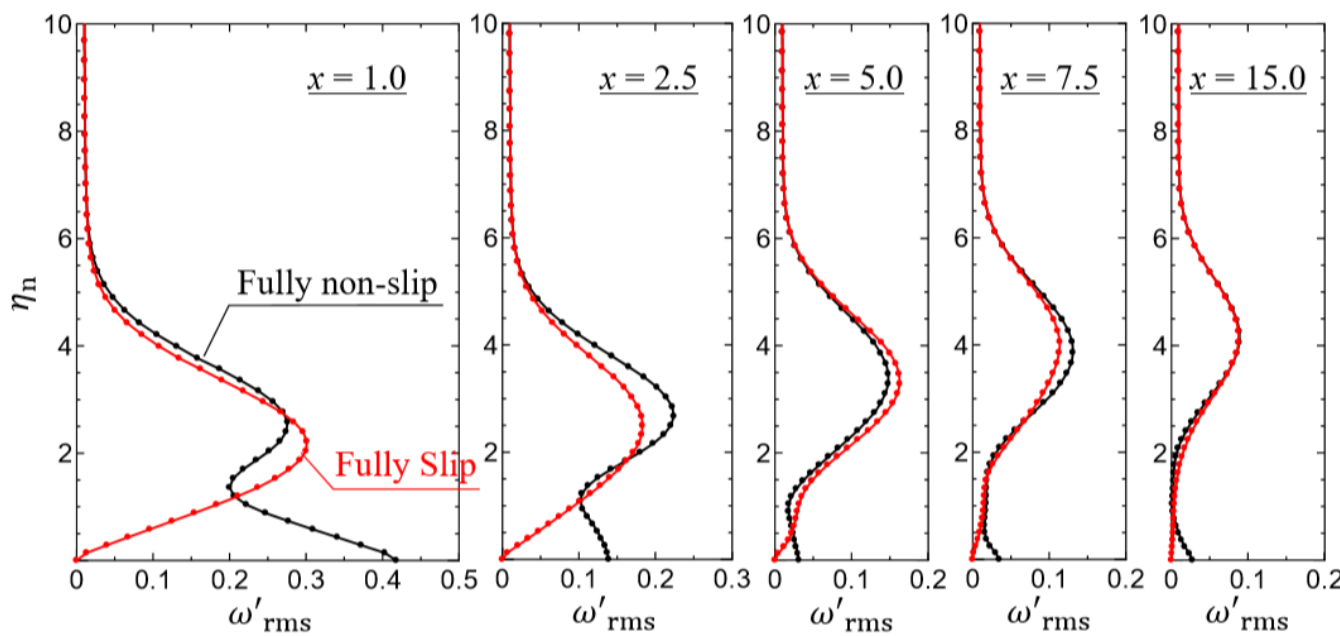

Fig. 8. Root-mean-square (RMS) of the vorticity fluctuations $\omega^{\prime}$ rms at $x=1.0,2.5,5.0,7.5$, and 15.0 under nonslip (Default: black) and slip conditions (Reference: red).

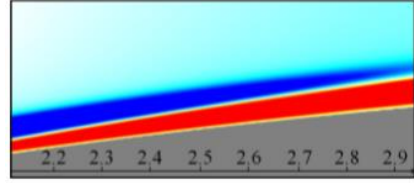

(a) Leading-edge

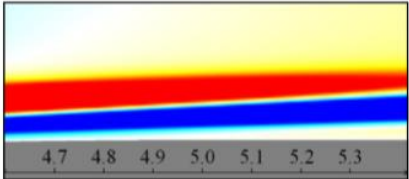

(b) Juncture

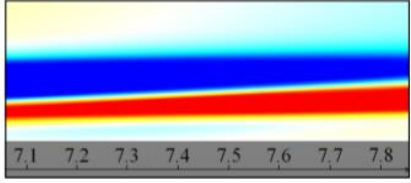

(c) Flat plate

Fig. 9. Enlarged views of the vorticity fluctuation pattern at three locations (slip condition).

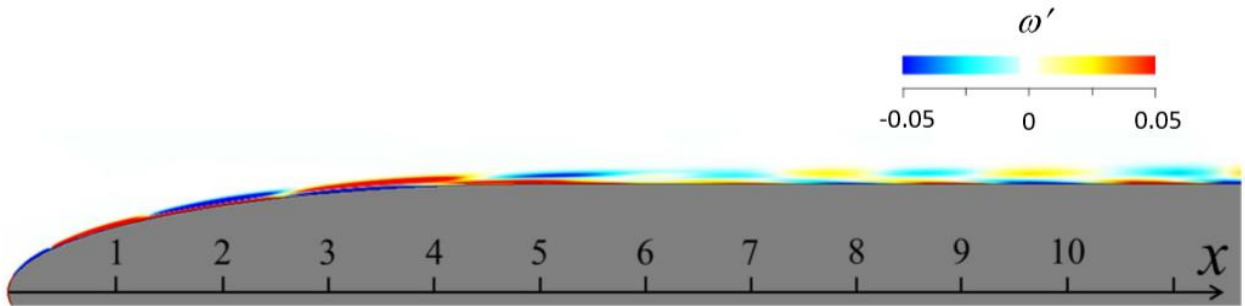

Fig. 10. Instantaneous distribution of vorticity fluctuation $\omega^{\prime}$ when vorticity fluctuations at the wall are given as a boundary condition.

originate from the freestream. Close-up views of vorticity distributions for the slip boundary condition case are shown in Fig. 9. Unlike the nonslip case shown in Fig. 5, no fluctuation pattern are observed in the near-wall region, implying that the T-S mode does not appear in the boundary layer.

\subsection{Role of Vorticity fluctuations Originating From the Wall Surface}

To study the role of the vorticity fluctuations originating from the wall surface, the following numerical experiment was performed.

1. First, the flow field with periodic vorticity fluctuations in the freestream a priori was computed using the ordinary nonslip condition, as shown in Fig. 4.

2. During the computation, the vorticity fluctuations at the wall were recorded at each time

3. A flow field without external vorticity fluctuations was computed by applying the recorded vorticity fluctuations as the Dirichlet boundary conditions at the wall surface.

Here, the base flow was assumed to be constant, as was in the previous sections.

The resulting instantaneous distribution of vorticity fluctuations once the fully periodic state is reached is shown in Fig. 10; close-up views of the leading edge, juncture, and flat surface sections are shown in Fig. 11. The vorticity fluctuations originating from the wall surface are primarily convected by the base flow and diffused by viscosity. Owing to the velocity gradient of the base flow inside the boundary layer, a pattern of elongated regions of positive and negative vorticity fluctuations were stacked one over another in the wall-normal direction and its sign changed at regular intervals in 
Table 1. Conditions of the supplied vorticity fluctuations at the wall surface.

\begin{tabular}{ccccc}
\hline Sections & Leading edge & Curved surface & Juncture & Flat surface \\
\hline $\begin{array}{c}\text { Fully nonslip } \\
\left(x_{f} \rightarrow \infty\right)\end{array}$ & nonslip & nonslip & nonslip & nonslip \\
\hline $\begin{array}{c}\text { Run I } \\
\left.x_{f}=1.0\right)\end{array}$ & slip & nonslip & nonslip & nonslip \\
$\begin{array}{c}\text { Run II } \\
\left(x_{f}=3.5\right)\end{array}$ & slip & slip & nonslip & nonslip \\
$\begin{array}{c}\text { Run III } \\
\left(x_{f}=5.5\right)\end{array}$ & slip & slip & slip & nonslip \\
\hline
\end{tabular}

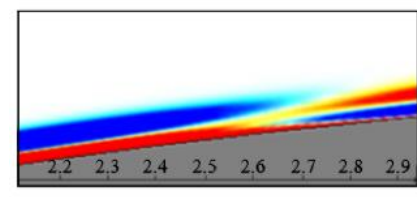

(a) Leading-edge

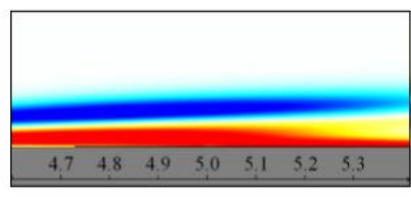

(b) Juncture

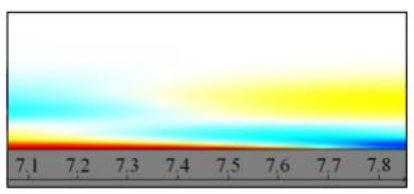

(c) Flat plate

Fig. 11. Enlarged view of Fig. 10 at three different streamwise locations. step.

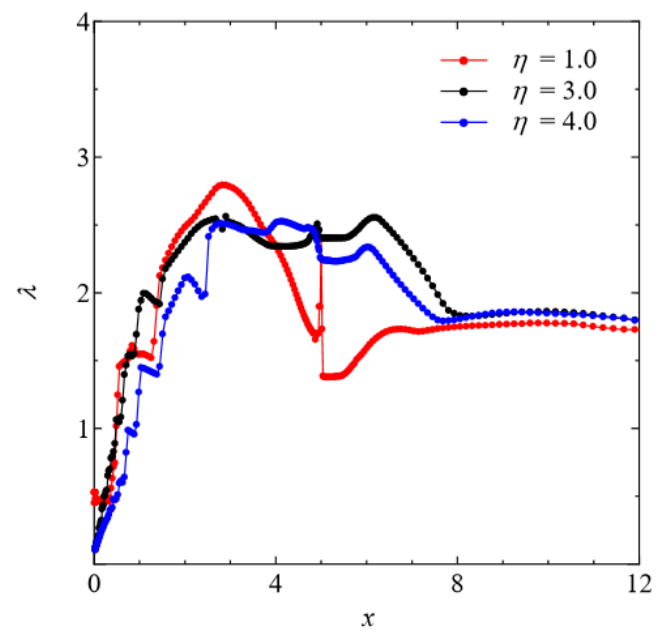

Fig. 12. Streamwise variation of the timeaveraged wavelength of the vorticity fluctuation patterns.

the streamwise direction in the downstream flat plate section.

Spatial variations of the wavelengths of these fluctuations $\lambda$ are presented in Fig. 12. The vorticity fluctuation was measured at each wall-normal distance $\eta_{\mathrm{n}}$, and the wavelength was estimated from the time-averaged distance between the two local maxima of $\omega^{\prime}$ in the streamwise direction over 11 cycles. The wavelengths increased near the leading edge and reached their maxima of $2.5 \sim 2.8$. The downstream of the juncture was at $x=5.0$; the wavelengths gradually approached $\lambda=1.7$ and converged at approximately $\mathrm{x}=8.0$ regardless of the wall-normal distance $\eta_{\mathrm{n}}$. The phase velocity of this wave was $34 \%$ of the freestream velocity, which agreed with the phase velocity of a typical T$\mathrm{S}$ wave. The wall-normal profile of the RMS of the streamwise velocity fluctuations measured at $x=10.0$ is shown in Fig. 13. The profile exhibits two peaks, which is a typical feature of T-S waves. Thus, the disturbance can be split into two groups: a convected disturbance which is restricted to the freestream and the outer boundary layer, and TS activity which only occurs near the wall. This finding is consistent with the numerical observation by Buter and Reed (1994).

\subsection{Numerical Experiments Applying Slip Boundary Condition to Limited Areas of Surface}

To compare the contributions of vorticity fluctuations supplied at various local areas along the wall surface, the supply of vorticity fluctuations at the wall were deliberately suppressed by applying the slip boundary condition to certain limited areas $\mathrm{x} \in\left[0, x_{f}\right]$ of the wall. Areas with slip and nonslip boundary conditions were smoothly connected at $x$ $=x_{f}$ using the following sigmoid function:

$f(x)=$

$$
\begin{cases}1 & \left(0 \leq x \leq x_{f}\right) \\ 1-\frac{1}{1+\exp \left\{-\beta\left(x-x_{f}-0.5\right)\right\}} & \left(x_{f} \leq x \leq x_{f}+1\right) \\ 1 & \left(x_{f}+\leq x \leq x\right)\end{cases}
$$

Here, the gain $\beta=10$ was used. The resulting profile of the filtering function, $f\left(x-x_{f}\right)$, is shown in Fig. 14. The slip boundary condition was applied for the region $x-x_{f}<0$ and the nonslip boundary condition was applied at $x-x_{f}>1$. The in-between region, $x_{f} \leq x \leq x_{f}+1$, was a buffer region.

The boundary layer was divided into four sections: the leading edge, curved surface, juncture, and flat surface. The slip and nonslip conditions were applied to selected sections, as shown in Table 1 . 

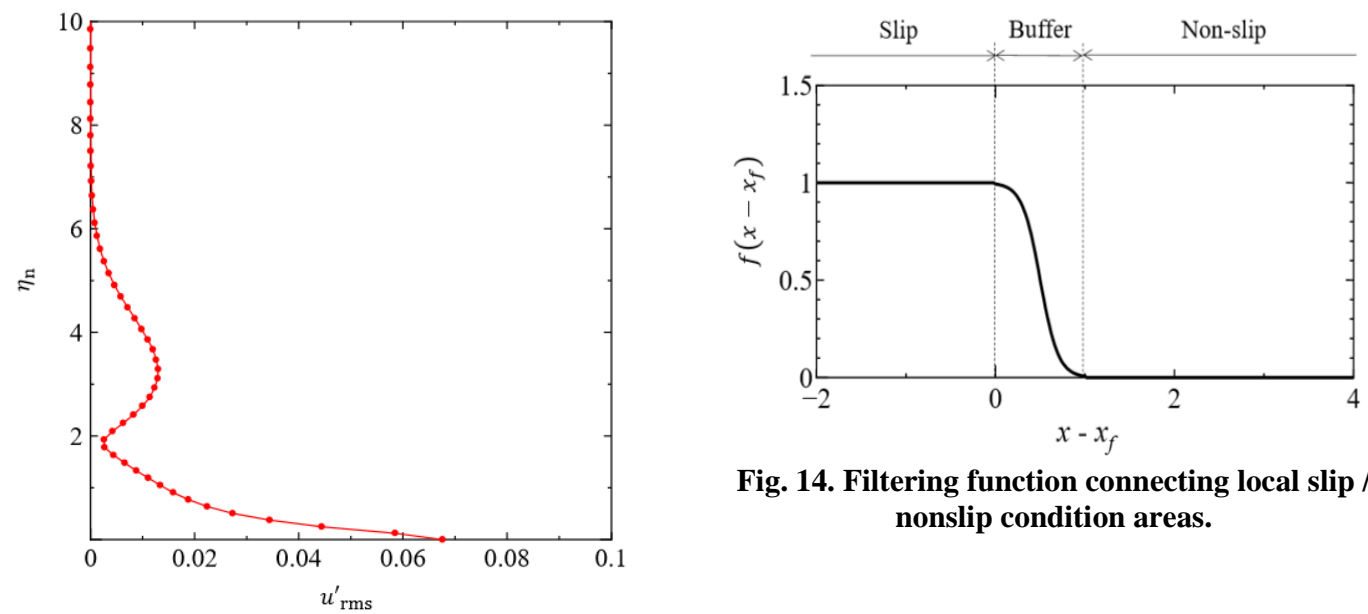

Fig. 14. Filtering function connecting local slip / nonslip condition areas.

Fig. 13. RMS of the velocity fluctuations $u^{\prime}$ at $\mathrm{x}=$ 10.0.

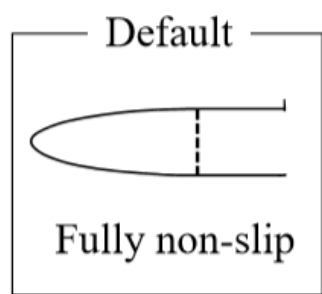

VS.

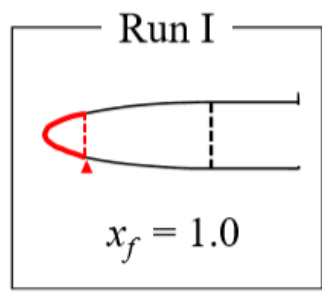

(a) Leading edge

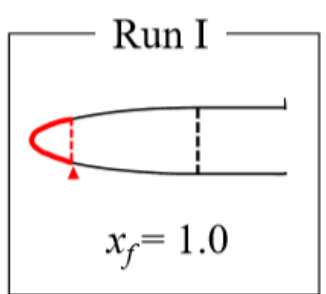

VS.

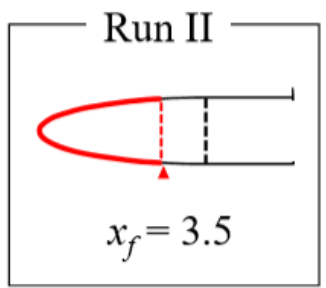

(b) Curved surface

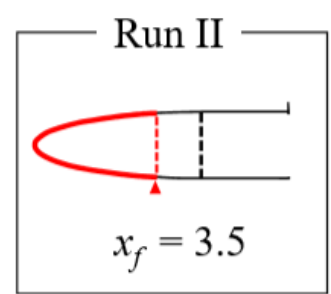

VS.

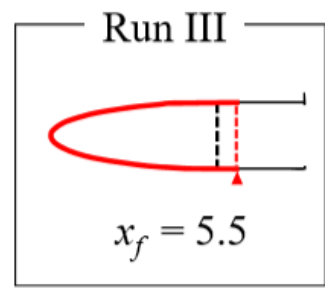

(c) Juncture

Fig. 15. Diagrams depicting three comparisons of dominant source of vorticity supply at the wall.

The starting points $x_{f}$ of the buffer region were at $x_{f}$ $=1.0,3.5$, and 5.5 for Runs I, II, and III, respectively. The corresponding buffer ranges were 1.0-2.0, 3.5-4.5, and 5.5-6.5, respectively. The location of the juncture between the curved and flat surfaces was at $\mathrm{x}=5.0$. The fully nonslip case $\left(\mathrm{x}_{\mathrm{f}}\right.$ $\rightarrow \infty$ ) was computed for comparison. Figure 15 shows the objective of Runs I, II, and III. The role of each section can be presented in the comparison between the two in which the target section has both the slip and nonslip boundary conditions.

First, the fully nonslip case and Run I is compared to understand the role of the leading edge section. Figure 16 shows the wall-normal direction profiles of the RMS of vorticity fluctuations measured at different streamwise stations, i.e., $x=1.0,2.5,5.0$, 7.5, and 15.0. The differences between the two cases reflect the role of the vorticity fluctuation generated at the surface of the leading edge section, corresponding to $0.0<x<1.5$. Only small differences can be observed in the downstream of $x$ $=5.0$, thereby indicating that the leading edge section is less important in the receptivity process.

The role of the curved surface section is presented in the comparison between Runs I and II. The results are plotted in Fig. 17. The contribution of the curved surface section, which is approximately 1.5 $<x<4.0$, is noteworthy. The difference between the two runs disappears at $x=15.0$ for $\eta_{\mathrm{n}}>1.0$; however, the vorticity fluctuation near the wall remains higher during Run II, which is approximately twice as large as Run I.

The effect of the juncture section appears when Runs II and III are compared. Here, the juncture between the curved and flat surfaces at $x=5.0$ is placed in the nonslip region during Run II and in the slip region during Run III. The juncture is known to exhibit high receptivity, as reported by Goldstein and Hultgren (1989). Figure 18 shows the 

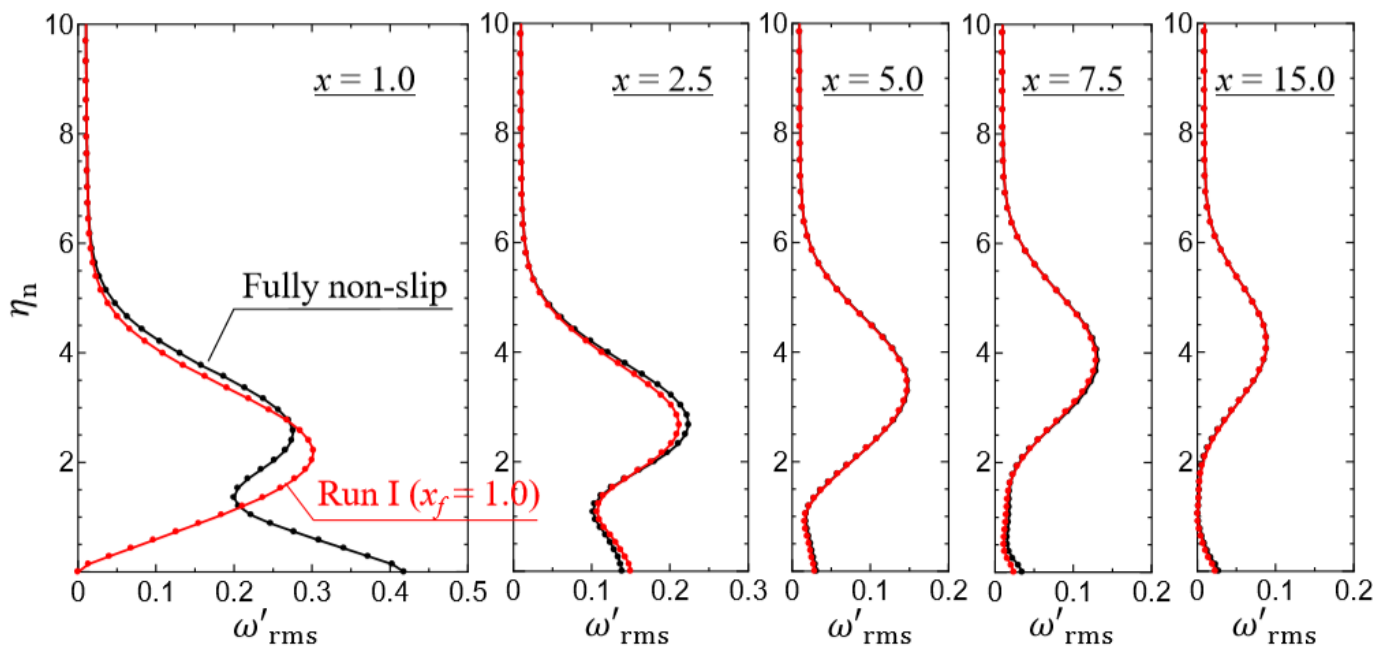

Fig. 16. RMS of the vorticity fluctuations $\omega^{\prime}$ rms at $x=1.0,2.5,5.0,7.5$, and 15.0 for comparing the fully nonslip run (black) and Run I (red).
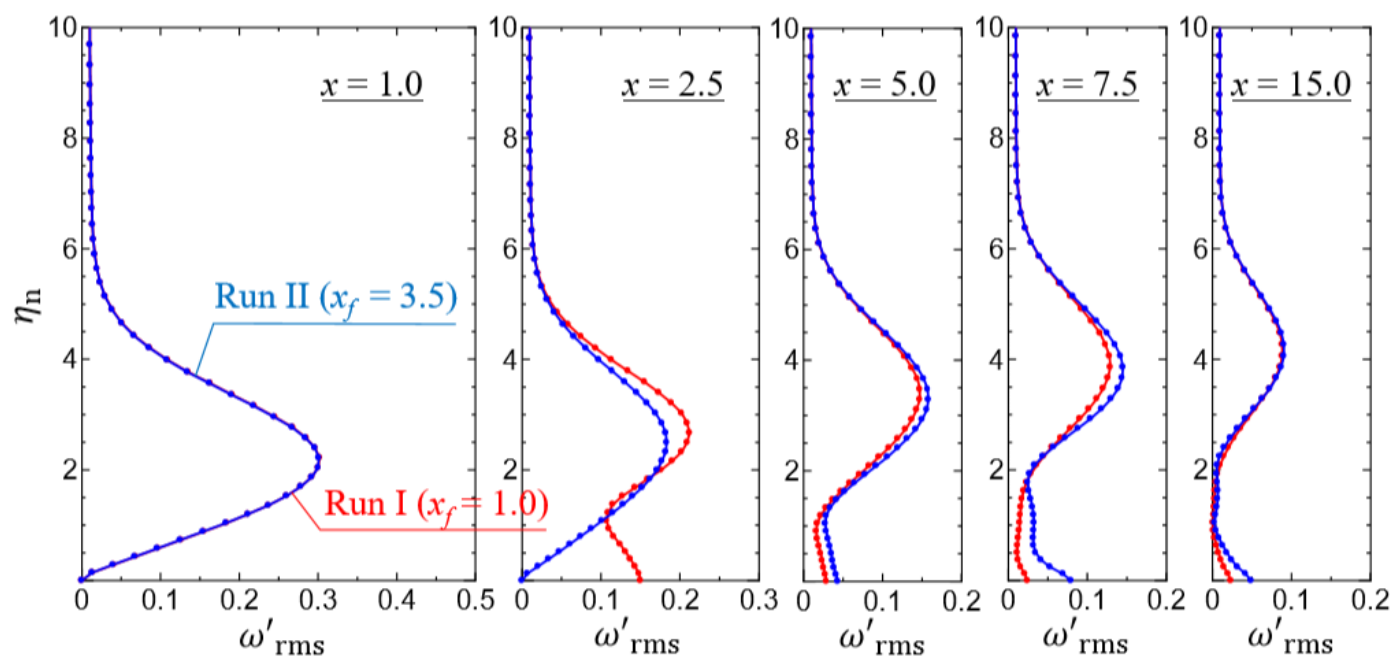

Fig. 17. RMS of the vorticity fluctuations $\omega^{\prime}$ rms at $x=1.0,2.5,5.0,7.5$, and 15.0 for Run $I\left(x_{f}=1.5\right.$, red $)$ and Run II $\left(x_{f}=4.0\right.$, blue).

comparison between these two cases. Although the difference between the two runs decreases at $\mathrm{x}=$ 15.0 for $\eta_{\mathrm{n}}>1.0$, some differences still remain close to the wall where the vorticity fluctuations in Run II exceed those in Run III.

The streamwise variation of the instantaneous velocity fluctuation $\mathrm{u}^{\prime}$ measured at $\eta_{\mathrm{n}}=1.0$ is shown in Fig. 19. Because the measured locations are close to the wall, the detected wavelengths agree with that of a typical T-S wave. Therefore, the velocity fluctuations observed are judged to be related to the T-S waves. It is noteworthy that the phases of the three cases, namely the fully nonslip, Run I, and Run II are all equal, whereas only the phase of Run III is different. The difference between the former three cases and Run III is that, the juncture part is under the slip condition in Run III. The fact that the phases of the former three cases match exhibits that the phase of the fluctuation is dominated by the vorticity fluctuation generated at the juncture section. The result agrees with preceding studies which showed the importance of the receptivity at the juncture (Goldstein and Hultgren 1989, Lin et al. 1992, Buter and Reed 1994). It should be noted that the largest amplitude appears in Run II followed by the fully nonslip case, while the amplitude of Run I is slightly smaller than that of the fully nonslip case. The difference between Runs I and II is owing to the effect of vorticity fluctuations generated at the curved surface section, which is deficient during Run II. Therefore, it is deduced that the vorticity fluctuations generated at that section contributed negatively to the total amplitude of velocity fluctuations observed in the fully nonslip case. Likewise, because the amplitude of Run I was slightly lower than that of the fully nonslip case, the vorticity fluctuations from the leading edge section contributed slightly but positively in terms of the generation of T-S waves, and short-wavelength velocity fluctuations occurred close to the wall. 

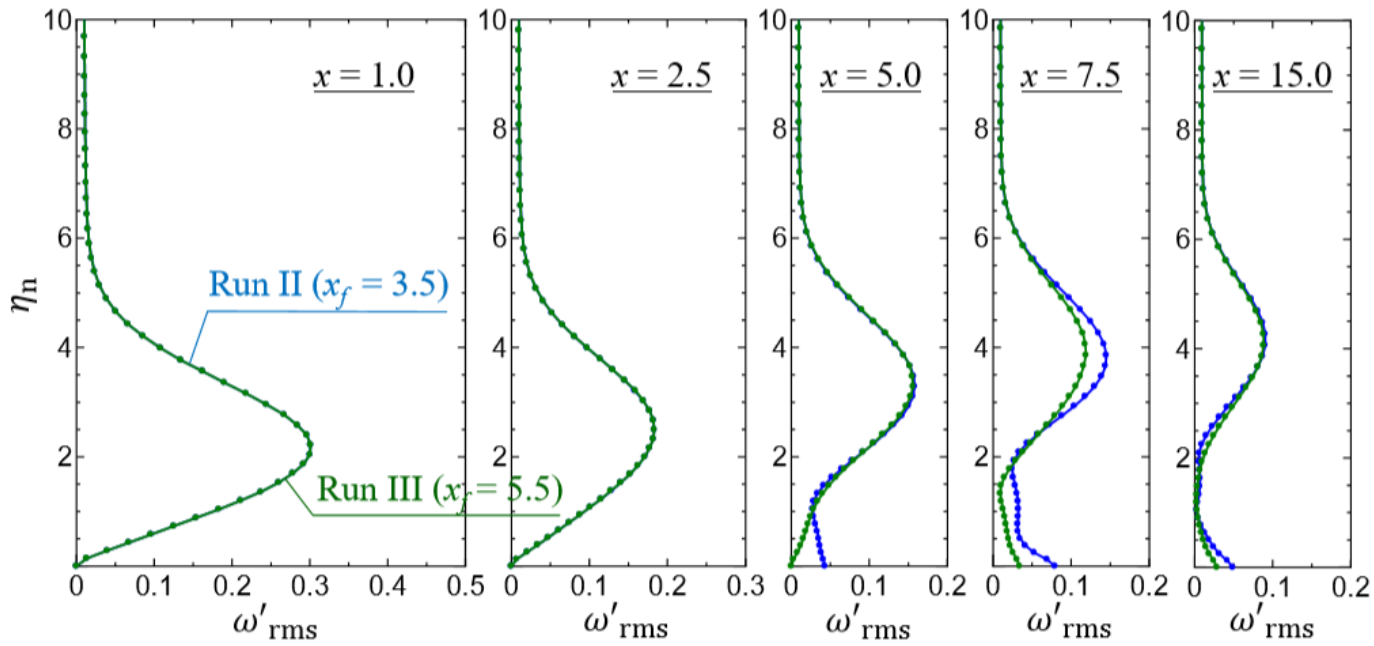

Fig. 18. RMS of the vorticity fluctuations $\omega^{\prime}$ rms at $x=1.0,2.5,5.0,7.5$, and 15.0 for Run II $\left(x_{f}=4.0\right.$, blue), and Run III $\left(x_{f}=6.0\right.$, green).

The results above suggest that the vorticity fluctuation generated at the juncture section is the most important, and that the vorticity fluctuations generated at other surface locations may enhance or weaken the fluctuation depending on their phase.

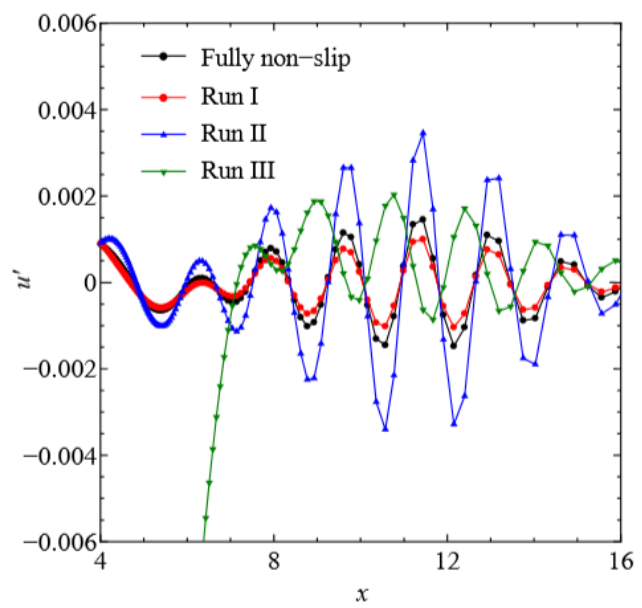

Fig. 19. Instantaneous streamwise velocity fluctuations $\mathrm{u}^{\prime}$ measured at $\eta_{\mathrm{n}}=\mathbf{1 . 0}$ for $\mathrm{t}=125$.

\section{CONCLUSIONS}

The receptivity of the Blasius boundary layer over a semi-infinite flat plate with an elliptic leading edge and of aspect ratio five was investigated by a direct numerical solution of two-dimensional NavierStokes equations. A time-periodic Lamb-Oseentype vorticity fluctuation was introduced into the freestream at the inlet boundary. The result of the computation where the slip condition was applied to the fluctuating component of velocity at the wall surface was compared with that of an ordinary computation using the nonslip condition. Furthermore, another numerical experiment was performed where no vorticity fluctuation was supplied from the freestream, while the prerecorded values of vorticities at the wall in response to the passage of convecting fluctuations were used as the wall vorticity boundary condition. It was shown that vorticity fluctuations in the boundary layer could be classified according to their wavelengths. Waves with longer wavelengths originated from the freestream, whereas waves with shorter wavelengths identified as T-S waves originated from the surface of the plate.

The slip boundary condition for the fluctuation component of vorticity was applied to the limited area of the wall surface to determine the part of the elliptic leading edge or flat plate that supplied vorticity fluctuations, thereby resulting in $\mathrm{T}-\mathrm{S}$ waves. The numerical results showed that the vorticity fluctuations originating from the juncture were the most crucial. Meanwhile, the vorticities supplied in the elliptic leading-edge surface contributed negatively to the amplitude of the vorticity fluctuations inside the boundary layer. The stagnation section did not contribute positively.

\section{REFERENCES}

Buter, T. A. and H. L. Reed (1994). Boundary layer receptivity to free-stream vorticity. Physics of Fluids 6(10) 3368-3379.

Dietz, A. (1999). Local boundary-layer receptivity to a convected free-stream disturbance. Journal of Fluid Mechanics 378.

Fransson, H. M., M. Matsubara, and P. H. Alfredsson (2005). Transition induced by freestream turbulence. Journal of Fluid Mechanics $527,1-25$.

Goldstein, M. E. and L. S. Hultgren (1989). Boundary layer receptivity to long-wave freestream disturbances. Annual Review of Fluid Mechanics 21(1) 137-166.

Hunt, J. C. R. and P. A. Durbin (1999). Perturbed vortical layers and shear sheltering. Fluid Dynamics Research 24(6) 375-404. 
Jacobs, R. G. and P. A. Durbin (1998). Shear sheltering and the continuous spectrum of the orrsommerfeld equation. Physics of Fluids 10 (8) 2006-2011.

Kerschen, E. J. (1990). Boundary layer receptivity theory. Applied Mechanics Reviews 43(55) S152-S157.

Kogan, M. N., V. G. Shumilkin, M. V. Ustinov and S. V. Zhigulev (2001). Response of boundary layer flow to vortices normal to the leading edge. European Journal of Mechanics B/Fluids 20(6) 813-820.

Lin, N., H. L. Reed and W. S. Saric (1992). Effect of leading edge geometry on boundarylayer receptivity to freestream sound. In Instability, Transition and Turbulence, 421-440.

Nishio, Y., M. Shigeta, S. Izawa and Y. Fukunishi (2013). Numerical study on leading-edge receptivity to freestream vertical vorticity. Journal of Fluid Science and Technology 8(1) 136-145.

Nishioka, M. and M. V. Morkovin (1986). Boundary-layer receptivity to unsteady pressure gradients: experiments and overview. Journal of Fluid Mechanics 171, 219-261.

Noro, S., M. Y., Shigeta, S. Izawa and Y. Fukunishi
(2014). Numerical study on receptivity of flatplate boundary layer to external disturbances. Theoretical Applied Mechanics 62, 3-12.

Noro, S., Y. Suzuki, M. Shigeta, S. Izawa and Y. Fukunishi (2013). Boundary layer receptivity to localized disturbances in freestream caused by a vortex ring collision. Journal of Applied Fluid Mechanics 6, 425-433.

Reed, H., E. Reshotko and W. S. Saric (2015). Receptiity: the inspiration of mark morkovin. AIAA Paper 2015-2471.

Reshotko, E. (2001). Transient growth: a factor in bypass transition. Physics of Fluids 13(5) 1067-10750.

Saric, W. S., H. L. Reed and E. J. Kerschen (2002). Boundary layer receptivity to freestream disturbances. Annual Review of Fluid Mechanics 34(1) 291-319.

Sengupta, A., S. V. K. and T. K. Sengupta (2019). Direct numerical simulation of vortex-induced instability for a zero-pressure-gradient boundary layer. Physical Review E 100 (3) 033118 .

VanderPlas, J. T. (2018). Understanding the lombscargle periodogram. The Astrophysical Journal Supplement Series 236(1) 1-28. 